\title{
UvCom1 Is an Important Regulator Required for Development and Infection in the Rice False Smut Fungus Ustilaginoidea virens
}

\author{
Xiaoyang Chen, ${ }^{1}$ Du Hai, ${ }^{1}$ Jintian Tang, ${ }^{1}$ Hao Liu, ${ }^{1}$ Junbin Huang, ${ }^{1}$ Chaoxi Luo, ${ }^{1}$ Tom Hsiang, ${ }^{2}$ and Lu Zheng ${ }^{1, \dagger}$ \\ ${ }^{1}$ The Key Lab of Plant Pathology of Hubei Province, Huazhong Agricultural University, Wuhan 430070, China \\ ${ }^{2}$ School of Environmental Sciences, University of Guelph, Guelph N1G 2W1, Canada \\ Accepted for publication 18 October 2019.
}

\begin{abstract}
Ustilaginoidea virens is an economically important biotrophic fungal pathogen that causes rice false smut worldwide. However, the regulatory mechanisms of smut ball formation under $U$. virens infection remain unclear. Here, we identified an important transcription factor, UvComl, from this pathogen, which regulates the formation of smut balls on rice spikelets. UvCom 1 contains two conserved internal repeat 1 (RPT) domains and is found only in fungi, with specific conservation in species of Basidiomycetes and Ascomycetes. UvCom1 protein N-or C-terminal fusion vectors both showed transactivation activity in yeast. Deletion of UvCom1 significantly affected the vegetative growth and conidiation of

U. virens. UvComl negatively regulated the responses to oxidative, osmotic, and cell wall stresses. Remarkably, UvComl was found to be essential for the formation of rice smut balls, and UvComl deletion mutants lost the ability to stably utilize nutrients from the rice host. UvComl was also highly expressed at the mycelial expansion stage. Transcriptomic analysis and quantitative real-time PCR revealed that UvCom 1 could affect the expression of genes significantly enriched in transmembrane transport. This study demonstrates that UvComl is a key transcription factor governing smut ball formation of this biotrophic fungus.
\end{abstract}

Rice false smut, which is caused by the pathogenic biotrophic fungus Ustilaginoidea virens (Cooke) Takah (teleomorph: Villosiclava virens), is one of the most devastating grain diseases in the majority of rice-growing areas of the world (Brooks et al. 2010; Guo et al. 2012; Qiu et al. 2019). As a grain disease, it causes severe losses of yield and quality (Jiang et al. 2009; Yang et al. 2012), and threatens human or animal health by producing cyclopeptide mycotoxins such as ustiloxins in false smut balls (Koiso et al. 1994; Nakamura et al. 1994; Zhou et al. 2012). U. virens usually infects rice by its conidia, which are generated on the surface of rice spikelets, after which the hyphae extend randomly and enter into the inner space of spikelets through small gaps between lemma and palea to colonize floral organs (Ashizawa et al. 2012; Song et al. 2016). In floral organs, the pathogen first attacks the filament, preventing pollen from maturing and also blocking ovary fertilization, and then invades the stigma and style to simulate fertilization, resulting in the hijacking of large amounts of nutrients for the formation of rice false smut balls (Song et al. 2016). The fungus activates the grain-filling genes by simulating the filling signal, then hijacks the nutrition from rice to form smut balls (Fan et al. 2015; Song et al. 2016; Tang et al. 2013).

Genome and transcriptome analyses of $U$. virens infection of rice have indicated that this fungus might contain many transcription factors that regulate its virulence (Zhang et al. 2014). However, only $U v P R O 1$ and $U v H o x 2$ in $U$. virens have been experimentally verified, and $U v P R O 1$ was found to play important roles in hyphal

†Corresponding author: L. Zheng; luzheng@mail.hzau.edu.cn

Funding: This work was supported by the National Key Research and Development Program (2017YFD0301400 and 2016YFD0300700), the Fundamental Research Funds for the Central Universities of China (2662018JC051) and the Special Technical Innovation of Hubei Province (2017ABA146).

*The $e$-Xtra logo stands for "electronic extra" and indicates that four supplementary figures and six supplementary tables are published online.

The author(s) declare no conflict of interest.

(c) 2020 The American Phytopathological Society growth, conidiation, stress response, and pathogenesis of $U$. virens ( $\mathrm{Lv}$ et al. 2016). UvHox2 regulates chlamydospore formation, conidiogenesis, and pathogenicity in $U$. virens (Yu et al. 2019). Here, we identified a new transcription factor, UvComl, in $U$. virens. Com1 was previously only identified in Magnaporthe oryzae, and deletion of MoComl resulted in reduced width and increased length of conidia; furthermore, the $\Delta$ Mocoml mutants exhibited significantly reduced virulence on rice and barley seedlings (Yang et al. 2010). Proteomic data have revealed that MoCom1 might have various effects on carbon and energy metabolism, lipid metabolism, melanin biosynthesis, structural organization of cells, and amino acid metabolism (Bhadauria et al. 2010). However, little has been known about the functions of Com 1 homologs in other fungi.

In this study, we found that the deletion of UvComl caused significant decreases in mycelial growth rate, conidial production, and tolerance to oxidative, osmotic, and cell wall integrity stresses. $\Delta U v$ com 1 mutants would normally infect rice filaments, stigmas, and styles and establish a nutritional relationship with rice floral organs but could not utilize large quantities of nutrients needed to form smut balls. RNA-sequencing (RNA-seq) analyses showed a large number of differentially expressed genes enriched in functions related to carbohydrate transport and metabolism, and membrane transport and activation, indicating that the deletion of UvCom1 should affect these functions, which resulted in a disorganized utilization of nutrients from rice. Taken together, our findings suggest that $U v C o m 1$ is a key regulator of transmembrane transport, and is critical for fungal growth and infection-related development.

\section{MATERIALS AND METHODS}

Fungal strains and growth conditions. The $U$. virens wildtype (WT) strain HWD-2, fully virulent on the rice cultivar Wanxian-98, was used in this study (Table 1). All transformed strains derived from the WT were cultured on potato sucrose agar (PSA) at $28^{\circ} \mathrm{C}$ under dark conditions. Seven-day-old mycelia collected from potato sucrose broth (PSB) shaken at $180 \mathrm{rpm}$ were used for isolation of fungal DNA and RNA, and the conidia 
separated from the culture were used for transformation via Agrobacterium-mediated transformation (ATMT), following Lv et al. (2016). Hygromycin B (final concentration of $200 \mu \mathrm{g} / \mathrm{ml}$ ) and G418 (final concentration of $500 \mu \mathrm{g} / \mathrm{ml}$ ) were used as selective markers for target gene deletion and complementation, respectively.

DNA and RNA manipulation, Southern blot and quantitative real-time PCR. Genomic DNA was extracted from vegetative hyphae using cetyltrimethylammonium bromide protocols. Southern blotting followed the protocol of Amersham Gene Images with Alkphos Direct Labeling and Detection System (GE Healthcare). Total RNA was extracted with the TRIzol reagent (Vazyme Biotech). To detect gene expression levels at different developmental stages, the total RNA of mycelia was collected from cultures grown for 7 days on PSB, or from infected rice spikelets at different days $(1,3,6,9,13$, or 20 days). Reverse-transcription assays were performed using a TransScript One-Step gDNA Removal and cDNA Synthesis SuperMix (TransGen Biotech). The $\beta$-tubulin gene (UV8b_900) was used as an internal control for quantitative real-time PCR (qRT-PCR). qRT-PCR was conducted using the TransStart Tip Green qPCR SuperMix (TransGen Biotech). Primer pair P7/P8 was designed for the detection of UvComl gene expression levels (Supplementary Table S1). All results were normalized by $\beta$-tubulin expression, and relative changes in gene expression levels were calculated by the comparative cycle threshold method (Applied Biosystems). The expression of genes associated with grain filling was the same as that used by Song et al. (2016) and the GAPDH gene was used to normalize the data, as documented previously (Fan et al. 2015; Song et al. 2016). For each gene, data were taken from three independent biological replicates.

Target gene deletion and complementation in $U$. virens. To generate $U v C o m l$ gene deletion mutants, approximately $1.5 \mathrm{~kb}$ of upstream and downstream flanking sequences of the gene were amplified with primers $\mathrm{F} 1 \mathrm{~F} / \mathrm{R}$ and $\mathrm{F} 2 \mathrm{~F} / \mathrm{R}$, respectively. The resulting PCR products were ligated with pGKO to generate the final deletion vector pGKO-UvCom 1 . The construct was inserted into the Agrobacterium tumefaciens strain EHA105, then transformed into conidia of the WT via the ATMT method, following Lv et al. (2016). Hygromycin-resistant transformants were isolated and subsequently screened by PCR with primers P1 and P2, then confirmed by RT-PCR and Southern blot.

For complementation assays, an approximately 4-kb fragment containing a $2 \mathrm{~kb}$ of native promoter region and a full-length UvCom1 gene-coding region was amplified from the genomic DNA of the WT using primers P11 and P12 and cloned into vector p3300neo. The EHA105 strain with the p3300neo-UvCom1 vector was transformed by coculturing with conidia of the mutant $\Delta U v$ com1-2 using the ATMT method (Lv et al. 2016). Transformants were selected on PSA supplemented with antibiotic G418 at $500 \mu \mathrm{g} / \mathrm{ml}$. Complementation strains were screened by conventional PCR with primers $\mathrm{P} 1$ and $\mathrm{P} 2$, then confirmed by

TABLE 1. Wild-type and mutant strains of Ustilaginoidea virens used in this study

\begin{tabular}{|c|c|c|}
\hline Strain & Description & Reference \\
\hline Wild type & Wild-type strain of $U$. virens HWD-2 & Jia et al. 2015 \\
\hline$\Delta$ Uvcom1-1 & UvCom1 deletion mutant of HWD-2 & This study \\
\hline$\Delta U v \operatorname{com} 1-2$ & UvCom 1 deletion mutant of HWD-2 & This study \\
\hline $\mathrm{C} \Delta U$ vcom $1-2$ & $\begin{array}{l}\text { UvCom } 1 \text { complementation strain } \\
\text { from } \Delta \text { UvCom } 1-2\end{array}$ & This study \\
\hline UvCom1-6 & $\begin{array}{l}\text { UvCom } 1^{1-659} \text {-green fluorescent protein } \\
\text { (GFP) subcellular localization strain }\end{array}$ & This study \\
\hline UvCom1-5 & $\begin{array}{l}\text { UvCom1 } 1^{1-210} \text {-GFP subcellular } \\
\text { localization strain }\end{array}$ & This study \\
\hline UvCom1-3 & $\begin{array}{l}\text { UvCom1211-659-GFP subcellular } \\
\text { localization strain }\end{array}$ & This study \\
\hline
\end{tabular}

RT-PCR. All primers used in this study are listed in Supplementary Table S1.

Vegetative growth, conidiation, and fungal morphology. To determine whether the function of UvComl was associated with the growth and morphogenesis of $U$. virens, the growth rate and colony morphology of $U$. virens transformants were investigated on PSA. For vegetative growth, mycelial plugs were transferred from 14-day-old PSA plates and grown on fresh PSA medium at $28^{\circ} \mathrm{C}$. After 14 days of incubation, the radial growth of vegetative mycelia was measured. Mycelial morphology of the strains was examined by Zeiss Axioplan light microscopy (Carl Zeiss). Because conidial production is an important process in many phytopathogenic fungi, conidiation of $U$. virens transformants was assessed in PSB. Strains were grown in PSB medium at $28^{\circ} \mathrm{C}$. After shaking at $180 \mathrm{rpm}$ for 7 days, the cultures were filtered through four layers of gauze, and conidial production was measured using a hemocytometer. Conidial morphology of the strains was examined by microscopy based on at least 500 conidia per feature. Each treatment was repeated three times.

Stress adaptation assays. To evaluate the role of $U v C o m 1$ in mediating $U$. virens adaptation to morphogenesis- or pathogenesisassociated stresses, the radial growth rates of the WT, $\Delta U v$ coml mutants, and complementation strains were compared on PSA containing the oxidative stress agent $\mathrm{H}_{2} \mathrm{O}_{2}$, osmotic stress agents $\mathrm{NaCl}$ or Sorbitol, cell-wall-disturbing agent sodium dodecyl sulfate (SDS), Congo red (CR), or Calcofluor white (CFW). Mycelial plugs
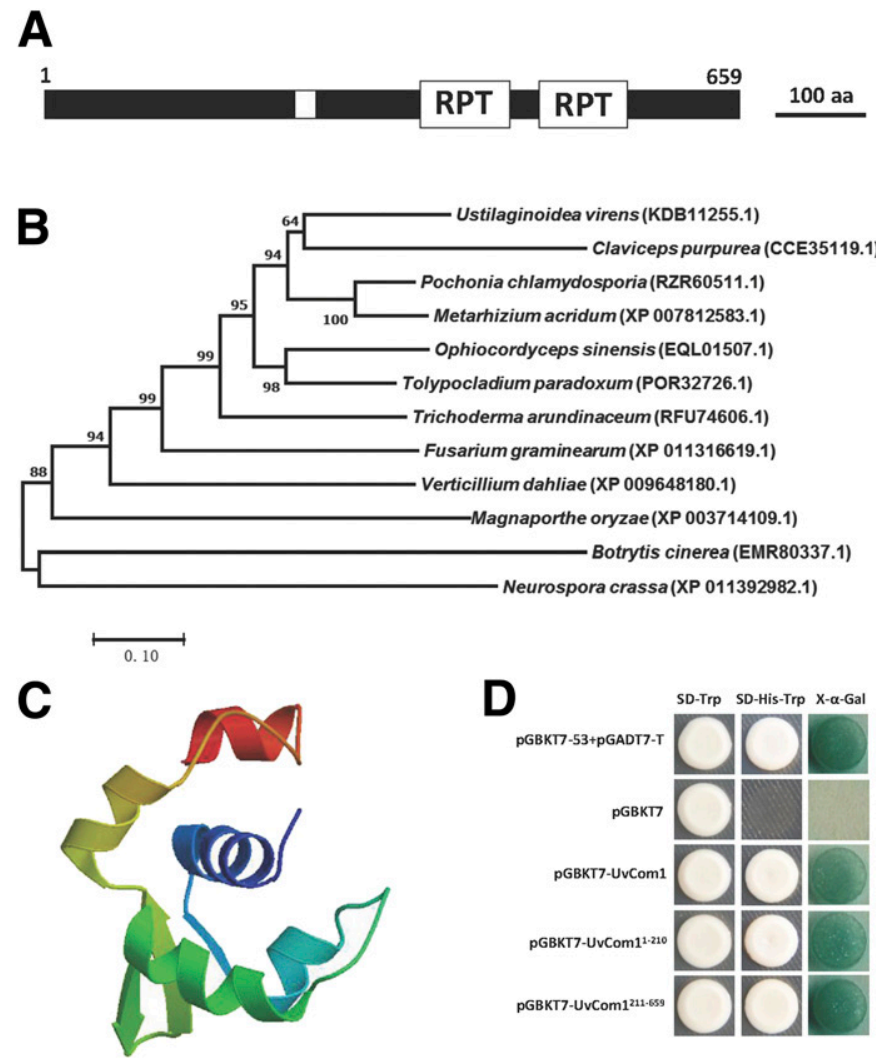

Fig. 1. Functional domain identification and phylogenetic tree. A, Predicted domain of internal repeat 1 (RPT) (box) of UvCom1 in Ustilaginoidea virens; $\mathrm{aa}=$ amino acids. B, Neighbor-joining tree of putative Com 1 homologs from 12 fungal genomes generated with MEGA 7.0. Numbers at branch nodes are bootstrap percentages out of 1,000 replicates. C, Three-dimensional (3D) structure model of UvCom 1 generated by Phyre 2 server. D, Transactivation analysis of UvCom1 and its deletion derivatives in yeast. The full-length UvCom1 and its deletion derivatives were fused with pGBKT7. The vectors pGBKT7 or pGBKT7-53 and pGADT7-T were expressed in yeast as negative or positive control. The $\beta$-galactosidase activity was measured with suitable $\mathrm{X}-\alpha-\mathrm{Gal}$. 
were inoculated onto PSA plates containing exogenous $0.3 \mathrm{M} \mathrm{NaCl}$, $0.5 \mathrm{M}$ Sorbitol, $0.03 \% \mathrm{H}_{2} \mathrm{O}_{2}, 0.03 \%$ SDS, CR at $120 \mu \mathrm{g} / \mathrm{ml}$, or $\mathrm{CFW}$ at $120 \mu \mathrm{g} / \mathrm{ml}$. Stress adaptation was assessed by measuring the colony diameter of 14-day-old cultures. Each treatment was repeated three times.

Virulence and plant infection assays. To investigate the function of $U v C o m l$ in $U$. virens infection, pathogenicity and plant infection assays of $\Delta U v \mathrm{vcom} 1$ mutants were conducted on a susceptible rice cultivar (Wanxian-98). The virulence of $U$. virens strains was tested based on the inoculation method of $\mathrm{Lv}$ et al. (2016). The WT, $\Delta U v$ coml mutant, and complementation strains were cultured in PSB medium at $28^{\circ} \mathrm{C}$. After shaking at $180 \mathrm{rpm}$ for 7 days, the cultures were homogenized in a blender, and rice plants were inoculated with $2 \mathrm{ml}$ of mycelial and spore suspensions using a syringe in the middle section of distal internodes at the eighth stage of panicle development. The treated rice plants were placed in a greenhouse at relative humidity of $95 \pm 5 \%$ and temperature of $28 \pm 2{ }^{\circ} \mathrm{C}$. The experiment was repeated three times.

After inoculation of rice panicles with $U$. virens, samples were taken 1 day and 6 days after inoculation, fixed with $2.5 \%$ (vol/vol) glutaraldehyde in $50 \mathrm{mM}$ phosphate buffer $(\mathrm{pH} \mathrm{7.2)}$ for 6 to $8 \mathrm{~h}$ at $4{ }^{\circ} \mathrm{C}$, then dehydrated with $30,50,70,80$, and $90 \%$ gradient alcohol

A
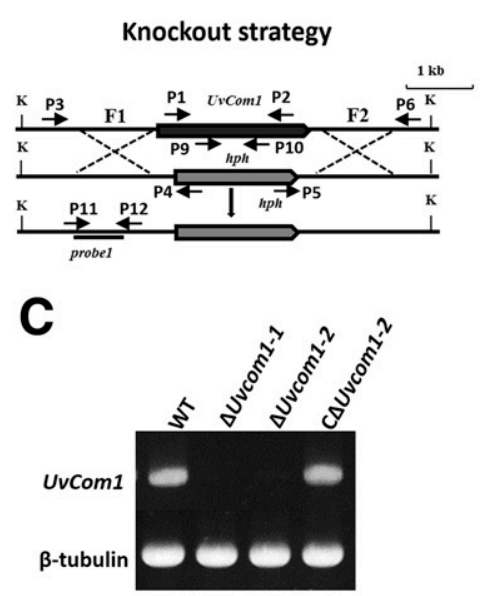

E

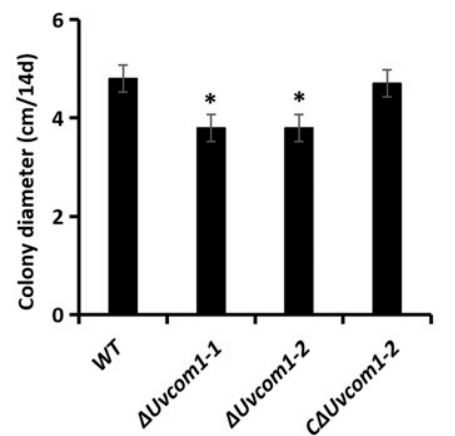

$\mathbf{F}$

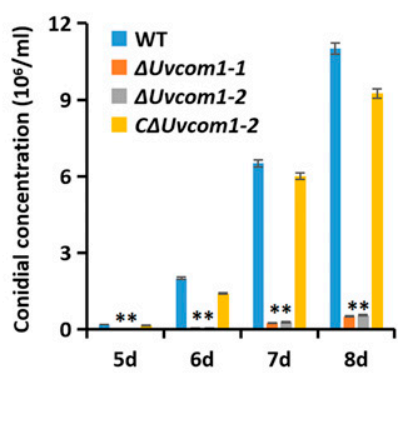

Fig. 2. Targeted gene deletion of UvComl in Ustilaginoidea virens. A, Knockout strategies of the $U v C o m 1$ gene and restriction sites for Southern blot at the UvComl genomic region. $\mathrm{K}=K p n \mathrm{I}$. B, Southern blot analysis of the wild type (WT) and $\Delta U v$ com 1 mutants. C, Real-time PCR analysis of the UvCom 1 mutants. D, Colony morphology of the UvCom 1 mutants on potato sucrose agar (PSA) after 14 days of darkness at $28^{\circ} \mathrm{C}$. E, Colony diameter on PSA after 14 days at $28^{\circ} \mathrm{C}$. Data were collected from three independent experiments for each treatment, and analyzed by Fisher's least significant difference test. Asterisks represent significant differences between the mutant and WT at $P=0.05$. F, Conidial production of the UvComl mutants in potato sucrose broth at $28^{\circ} \mathrm{C}$ and $180 \mathrm{rpm}$ for 7 days. and acetone. The samples were critical point dried, mounted on stubs, sputter coated with gold-palladium, and viewed using a JEOL JSM-6390LV scanning electron microscope operating at $10 \mathrm{kV}$.

Generation of green fluorescent protein fusion constructs and subcellular localization analysis. A full-length $U v C o m 1$ gene-coding region with native promoter was amplified from the genomic DNA of the WT using primers UvCom1 ${ }^{1-659} \mathrm{~F}$ and R (Supplementary Table S2), and cloned into vector p3300neogreen fluorescent protein (GFP). A full-length UvComl genecoding region was cloned into vector p3300neo-RP27-GFP. The sequences of the two exons were ligated into vector p3300neoRP27-GFP to obtain the UvCom11-210_ or UvCom1211-659-GFP fusion construct under the control of the RP27 promoter. The EHA105 strain with the GFP fusion vectors was transformed using ATMT by coculturing with the conidia of the $\Delta U v \operatorname{vom} 1-2$ or WT. The transformants were selected on PSA supplemented with antibiotic G418 at $500 \mu \mathrm{g} / \mathrm{ml}$. For subcellular localization analysis of UvCom1, the GFP signal was observed by a Zeiss LSM 510 Meta confocal microscope (Carl Zeiss), and imaged using a 488-nm diode laser for excitation and a 500- to 550-nm band-pass emission filter.

Transactivation activity assay in yeast. To examine transcriptional activity of UvCom1, yeast one-hybrid assays were performed using the Matchmaker pGBKT7 (Clontech) and the full length of $U v C o m 1$ was amplified and inserted into pGBKT7 for construction of vector pGBKT7-UvCom $1^{1-659}$. To further determine which region of the protein is involved in transcription activation, the two exons of $U v C o m 1$ were separately amplified and inserted into pGBKT7. These two constructed vectors were termed pGBKT7-UvCom1 1-210 (N-terminal fusion vector) and pGBKT7UvCom1211-659 (C-terminal fusion vector). The interaction between pGBKT7-53 and pGADT7-T was taken as the positive control and pGBKT7 was taken as the negative control. The three constructs

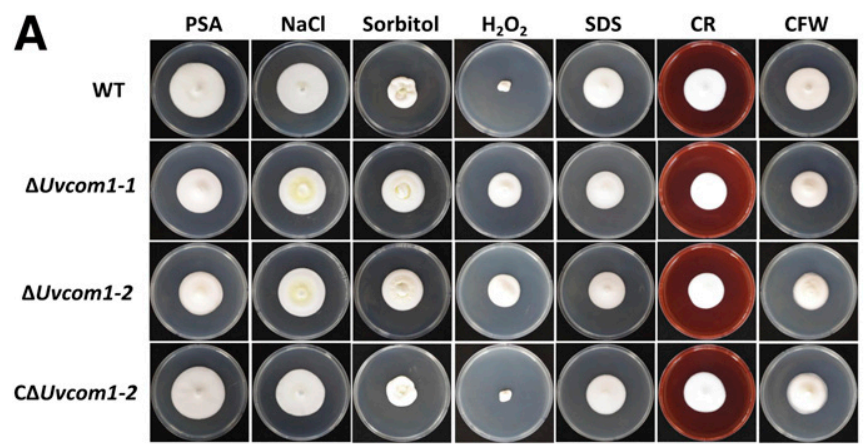

B

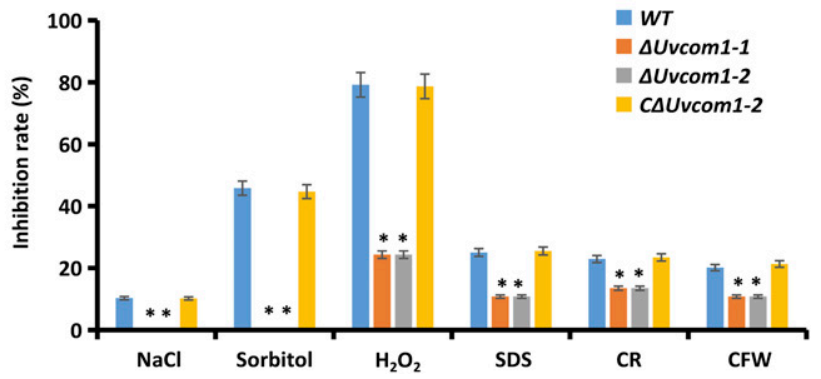

Fig. 3. Sensitivity of $U v$ Com 1 deletion mutants to various stresses. A, Growth on potato sucrose agar (PSA) with various amendments- $0.3 \mathrm{M} \mathrm{NaCl}, 0.5 \mathrm{M}$ Sorbitol, $0.03 \% \mathrm{H}_{2} \mathrm{O}_{2}, 0.03 \%$ sodium dodecyl sulfate (SDS), Congo red (CR) at $120 \mu \mathrm{g} / \mathrm{ml}$, or Calcofluor white (CFW) at $120 \mu \mathrm{g} / \mathrm{ml}$ - after 14 days at $28^{\circ} \mathrm{C}$. $\mathbf{B}$, Inhibition of colony growth by various stress inducers for $U v C o m 1$ mutants and the wild type (WT). Data were collected from three independent experiments for each treatment and analyzed by Fisher's least significant difference test. Asterisks represent significant differences between the mutant and WT at $P=0.05$. 
were separately transformed into the yeast strain $\mathrm{Y} 2 \mathrm{H}$. All transformants were isolated with SD-Trp, and the $\beta$-galactosidase activity was measured on SD-Trp-His medium with suitable $\mathrm{X}-\alpha$-Gal (Clontech). All primers used for UvCom 1 transactivation activity assay in the yeast are presented in Supplementary Table S1.

RNA sequencing. To investigate the regulatory mechanism of UvComl, deep transcriptome sequencing was used to compare the gene expression profiles between the WT and $\Delta U v$ com 1-2 mutant. Total RNA was extracted from 7-day-old mycelia of the two strains using TRIzol reagent, and each strain set three biological replicates. RNA-seq transcriptome libraries were prepared with the TruSeq RNA Sample Preparation Kit (Illumina) using $5 \mu \mathrm{g}$ of total RNA. Briefly, messenger RNA was first isolated according to a polyA selection method by oligo(dT) beads, then fragmented by fragmentation buffer. Next, double-stranded cDNA was synthesized using a SuperScript double-stranded cDNA Synthesis Kit (Invitrogen) with random hexamer primers (Illumina). Then, the synthesized cDNA was subjected to end repair, phosphorylation, and ' $\mathrm{A}$ ' base addition according to Illumina's library construction protocol. Libraries were size selected for cDNA target fragments of 200 to $300 \mathrm{bp}$ on $2 \%$ low-range ultra agarose followed by PCR. After being quantified by TBS380, paired-end RNA-seq libraries were sequenced on the Illumina HiSeq4000 SBS Kit (300 cycles) with 150 -bp paired-end reads. The library preparation and Illumina sequencing were performed by Shanghai Majorbio Bio-Pharm Technology Co., Ltd.

Accession numbers. The RNA-seq data used in this study have been deposited in the NCBI Sequence Read Archive database with accession numbers SRR10101940 to SRR10101945.

Bioinformatics analysis. The full sequence of $U v C o m 1$ was downloaded from the $U$. virens database (https://www.ncbi.nlm. nih.gov/nuccore/JHTR00000000). Protein-conserved domains were predicted with SMART (http://smart.embl-heidelberg.de/). The three-dimensional (3D) structure modeling of proteins was
A
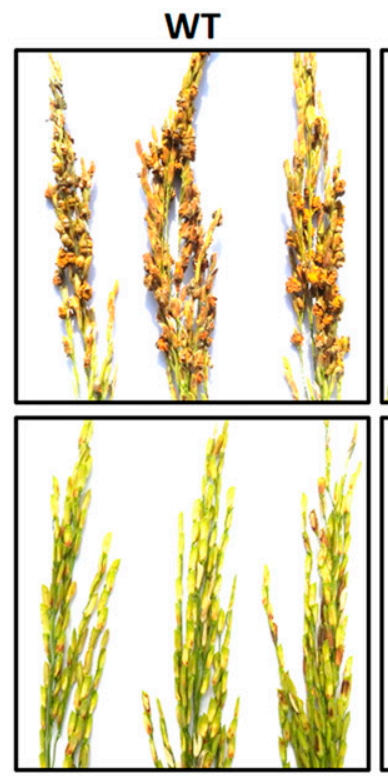

$\Delta U$ vcom1-2
$\Delta U v \operatorname{com} 1-1$
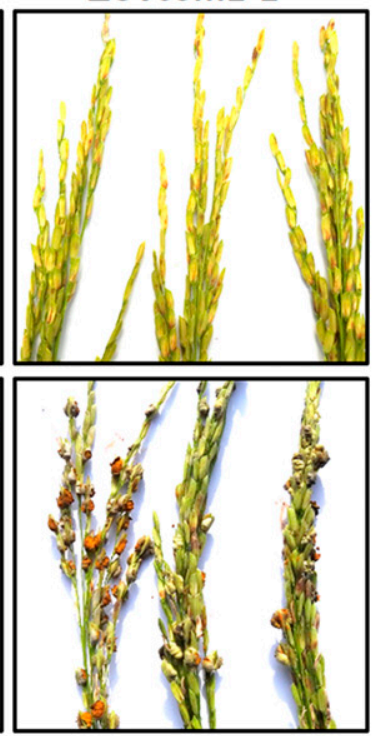

CAUvcom1-2
B

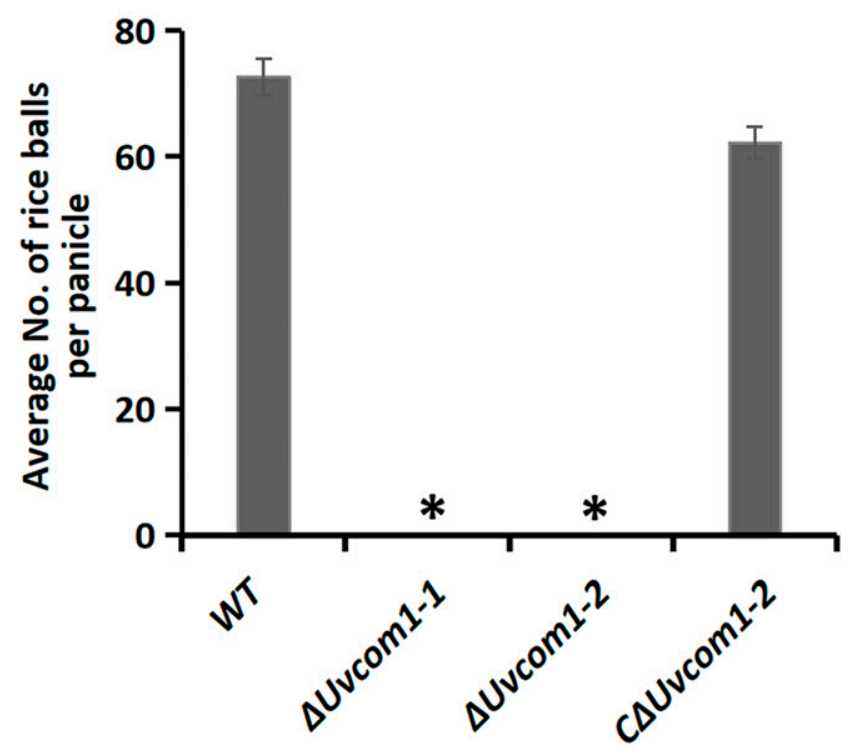

C
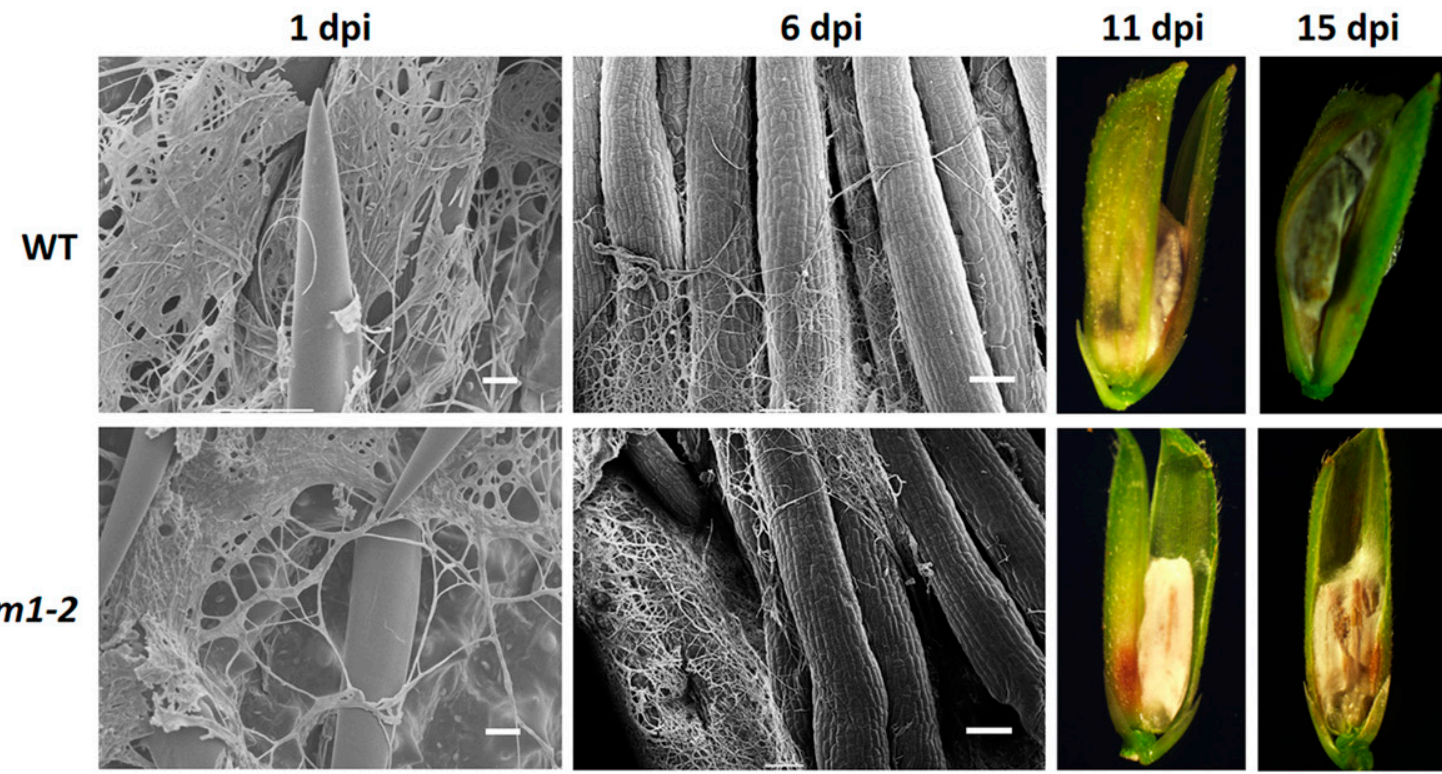

18 dpi
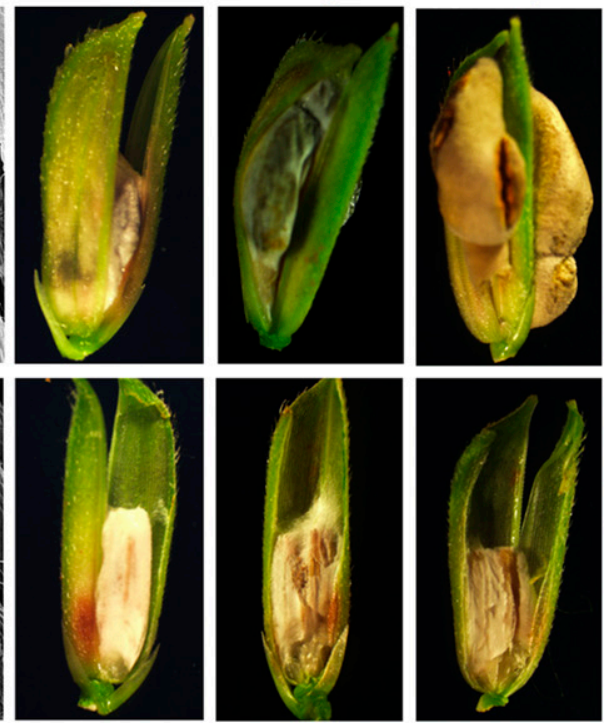

Fig. 4. Virulence assay and infection development of $U v \operatorname{Com} 1$ deletion mutants on rice spikelets. A, Virulence of the wild type (WT) and $U v C o m 1$ mutants at 21 days postinoculation (dpi) on rice cultivar Wanxian-98. B, Average number of smut balls per panicle. Data were collected from three independent experiments for each treatment and analyzed by Fisher's least significant difference test. Asterisks represent significant differences between the mutant and WT at $P=0.05$. $\mathbf{C}$, Infection progress of rice spikelets by the WT and $\Delta U v$ coml. Scale bar $=50 \mu \mathrm{m}$. 
carried out using the Protein Homology/analog Y Recognition Engine v 2.0 (Phyre2) program (http://www.sbg.bio.ic.ac.uk/ $\sim$ phyre $2 / \mathrm{html} /$ page.cgi?id=index). Com 1 homologous protein sequences from 11 other organisms were obtained from the NCBI and their accession numbers are listed as follows: Metarhizium acridum (XP007812583.1), Claviceps purpurea (CCE35119.1), Fusarium graminearum (XP011316619.1), Magnaporthe oryzae (XP003714109.1), Neurospora crassa (XP011392982.1), Botrytis cinerea (EMR80337.1), Pochonia chlamydosporia (RZR60511.1), Ophiocordyceps sinensis (EQL01507.1), Tolypocladium paradoxum (POR32726.1), Trichoderma arundinaceum (RFU74606.1), and Verticillium dahliae (XP009648180.1). After sequence alignments with ClustalW v2.1, the phylogenetic tree was generated by the Mega7.0 Beta program using the neighbor-joining algorithm, and the bootstrap analysis with 1,000 replicates was performed to test the confidence of topology.

For RNA-seq data analysis, the raw paired-end reads obtained from sequencing were trimmed and quality controlled by SeqPrep (https://github.com/jstjohn/SeqPrep) and Sickle (https://github. $\mathrm{com} /$ najoshi/sickle) with default parameters. Clean reads were then separately aligned to the reference genome with orientation mode using TopHat software (version 2.0.0; http://tophat.cbcb.umd. edu/) (Trapnell et al. 2009). The library construction quality and sequencing quality of each sample are shown in Supplementary Tables S2 and S3. Based on fragments per kilobase of exon per million mapped reads, the expression level of each transcript was calculated. The RSEM software (http://deweylab.biostat.wisc.edu/ $\mathrm{rsem} /$ ) was used to quantify gene abundances (Li and Dewey 2011). $\mathrm{R}$ statistical package software edgeR (Empirical Analysis of Digital Gene Expression in R; (http://www.bioconductor.org/packages/ 2.12/bioc/html/edgeR.html) (Robinson et al. 2010) was utilized for differential expression analysis. Functional-enrichment analyses, including gene ontology (GO) and Kyoto Encyclopedia of Genes and Genomes (KEGG), were performed to identify which differentially expressed genes (DEGs) were significantly enriched in GO terms and metabolic pathways at Bonferroni-corrected $P$ value $\leq$ 0.05 compared with the whole-transcriptome background. GO functional enrichment and KEGG pathway analysis were carried out by Goatools (https://github.com/tanghaibao/Goatools) and KOBAS (http://kobas.cbi.pku.edu.cn) (Xie et al. 2011).

Statistical analysis. Data were subjected to analyses of variance using SPSS 13.0 software (SPSS Inc.). Mean comparisons were conducted using Fisher's least significant difference test $(P=0.05)$.

\section{RESULTS}

Identification and characterization of $U v C o m 1$ in $U$. virens. Com 1 was identified through homology search of the $U$. virens genome database by using the BLASTP algorithm with a sequence of Com 1 from $M$. oryzae as query, and named UvCom 1 in this study. The $U v C o m 1$ gene contained two exons, and its exonintron structure was verified by RT-PCR (data not shown). Functional domain analysis demonstrated that UvComl contains two conserved internal repeat 1 (RPT) domains (Fig. 1A). Phylogenetic analysis of Coml homologs from different fungi revealed that UvCom 1 is the most similar to the homolog from C. purpurea and is specifically conserved in species of the divisions Basidiomycetes and Ascomycetes (Fig. 1B). The $3 \mathrm{D}$ structure modeling showed that UvCom1 protein has putative helix-loop-helix structures (Fig. 1C). In transactivation activity assays, yeast transformants containing vector pGBKT7-UvCom11-659, pGBKT7-UvCom11-210-N, or pGBKT7-UvCom1211-659-C could grow normally on SDTrp-His plates and colonies appeared blue (Fig. 1D). These results indicate that $U v C o m l$ is a transcription factor, and both $\mathrm{N}$ terminal and C-terminal domains of the protein are involved in transcription activation.
UvCom 1 deletion significantly affected vegetative growth and conidiation of $\boldsymbol{U}$. virens. In total, 20 transformants were obtained from the UvComl knock-out library, which was constructed by homologous recombination strategy (Fig. 2A), and eight independent deletion mutants with similar phenotypes were identified by PCR analyses (Supplementary Fig. S1), with primers listed in Supplementary Table S1. We then randomly selected two UvComl deletion mutants, $\Delta U v \operatorname{com} 1-1$ and $\Delta U v \operatorname{com} 1-2$, to further confirm the UvComl gene deletion event by Southern blot and RT-PCR analyses (Fig. 2B and C). The Southern blotting showed that an approximately $7.2-\mathrm{kb}$ band was detected in the mutants $\Delta U v$ com $1-1$ and $\Delta U v \operatorname{com} 1-2$, in contrast with an approximately $9.8-\mathrm{kb}$ band in the WT, indicating that the $U v C o m l$ gene was deleted in these two mutants. RT-PCR results demonstrated that UvComl was not expressed in the selected two mutants. Hence, the deletion mutants $\Delta U v$ com 1-1 and $\Delta U v$ com 1-2, serving as two independent repetitions, were further chosen for functional characterization of UvCom1. In a complementation assay, the complemented transformant $\mathrm{C} \Delta U$ vcom1-2 recovering normal phenotypes was confirmed by RT-PCR and the results showed that the UvComl gene could be expressed in mycelia of C $\Delta U$ vcoml-2 (Fig. 2C).

Compared with the WT, the mycelial growth rate of $\Delta U v$ coml mutants was significantly reduced (Fig. 2D and E) $(P<0.05)$. We also observed the mycelial morphology under microscopy but found no apparent differences in cells and cellular structures between $\Delta U v$ com 1 mutants and the WT (Supplementary Fig. S2A). Conidial production was also significantly reduced in $\Delta U v$ coml mutants (Fig. 2F) $(P<0.05)$ but no morphological defects were observed in the conidia of $\Delta U v$ com 1 mutants (Supplementary Fig. $\mathrm{S} 2 \mathrm{~B}$ ). These results indicated that $U v C o m 1$ plays important roles in the vegetative growth and conidiation of $U$. virens.

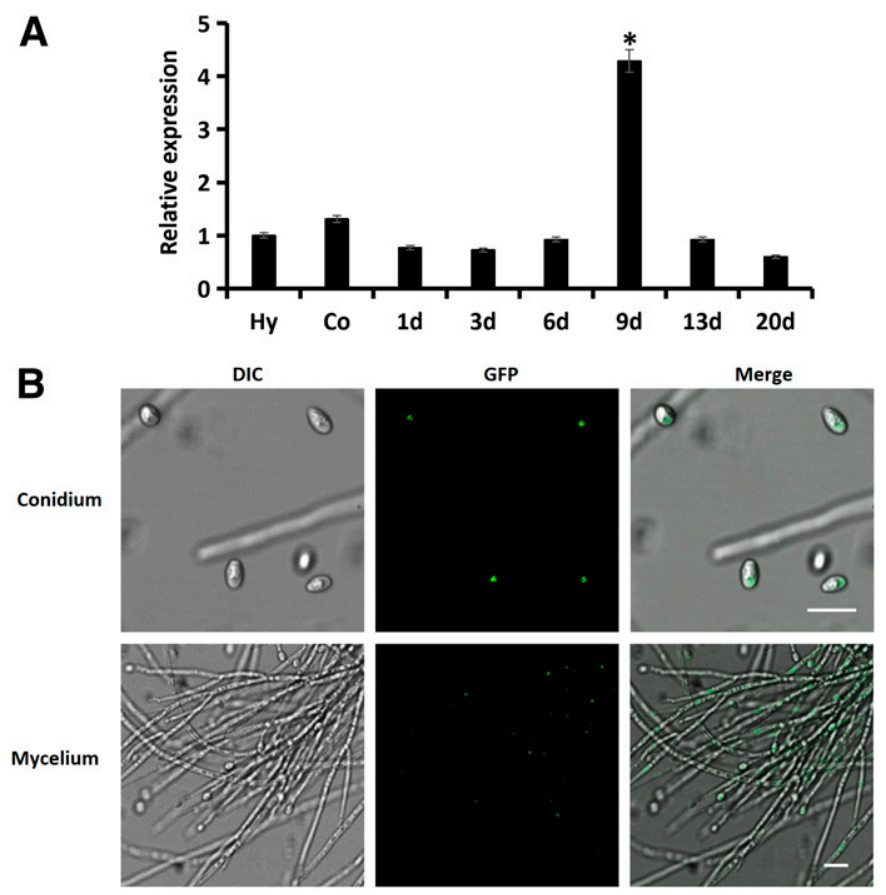

Fig. 5. Expression and subcellular localization of UvCom1 in Ustilaginoidea virens. A, Expression of UvCom1 relative to $\beta$-tubulin in conidia (Co) and hyphae (Hy) in potato sucrose broth (PSB) and at different infection stages on rice ( 1 to 20 days) by quantitative real-time PCR. Data were collected from three independent experiments for each treatment and analyzed by Fisher's least significant difference test. Asterisks indicate significant differences compared with Hy $(P=0.05)$. B, Subcellular localization of UvCom $1^{211-659}$ in $U$. virens. Vegetative hyphae and conidia of the transformant expressing UvCom1 $1^{211-659}$-GFP were observed under epifluorescence microscopy. DIC = differential interference contrast, GFP $=$ green fluorescent protein, and scale bar $=20 \mu \mathrm{m}$. 
UvCom1 negatively regulated responses to oxidative, osmotic, and cell wall stresses. Among all tested stress chemicals, $0.3 \mathrm{M} \mathrm{NaCl}$ and $0.5 \mathrm{M}$ sorbitol showed no inhibitory effect on the $\Delta U v$ coml mutants but significantly inhibited the mycelial growth of the WT $(P<0.05)$. Furthermore, the stress agents of $0.03 \% \mathrm{H}_{2} \mathrm{O}_{2}, 0.03 \% \mathrm{SDS}, \mathrm{CR}$ at $120 \mu \mathrm{g} / \mathrm{ml}$. or CFW at $120 \mu \mathrm{g} / \mathrm{ml}$ had fewer significant inhibitory effects on the $\Delta U v$ com 1 mutants than on the WT (Fig. $3 \mathrm{~A}$ and B) $(P<0.05)$. These results suggested that $U v C o m 1$ may negatively regulate the responses to oxidative or osmotic stresses and cell wall integrity of $U$. virens.

UvCom 1 was essential for fungal pathogenicity. In pathogenicity assays, after 21 days postinoculation (dpi), a typical symptom of yellow smut balls was observed on rice spikelets infected by the WT and complementation strains, whereas the $\Delta U v$ com 1 mutants failed to produce such a symptom (Fig. $4 \mathrm{~A}$ and $\mathrm{B}$ ).

For all tested strains, at $1 \mathrm{dpi}$, hyphae were found to be elongated and extended along the surface of spikelets. At $6 \mathrm{dpi}$, hyphae were observed on the surfaces of filaments of rice colonized by the WT and $\Delta U v \operatorname{com} 1$ mutants. Until $18 \mathrm{dpi}$, rice grains were not found to be forming the ball-like colonies by $\Delta U v$ coml mutants, whereas infection by WT and complementation strains normally led to the formation of rice smut balls (Fig. 4C). These results indicated that $\Delta U v \operatorname{com} 1$ mutants could establish a nutritional relationship with rice spikelets but could not form smut balls.

UvCom 1 was highly expressed at the mycelial expansion stage in rice spikelets. The gene expression profile of $U v C o m l$ at different developmental stages of $U$. virens during pathogenesis was examined. In comparison with the vegetative-mycelial stage, the expression of UvCom 1 was significantly increased at the stage of mycelial colonization in rice spikelets at 9 dpi (Fig. 5A) $(P<0.05)$, suggesting that $U v C o m 1$ might be involved in mycelial expansion to form rice smut balls during $U$. virens infection.
UvCom1211-659 determines the nuclear localization of UvCom 1 protein. In the subcellular protein localization assay of UvCom1, none of the transformants had detectable GFP signals in vegetative hyphae or conidia under control of its native promoter. To determine whether the native promoter of UvCom 1 affected the accumulation of GFP signals in the transformants, we also generated transformants with the p3300neo-RP27-UvCom1-GFP C-terminal fusion construct under the control of the RP27 promoter. However, GFP signals were not detected in vegetative hyphae and conidia of any transformants. To test whether the GFP-UvCom1 C-terminal fusion interfered with the detection of GFP, we subsequently generated transformants with p3300neo-RP27-GFPUvCom1 N-terminal fusion constructs. All transformants could recover normal phenotypes but GFP signals were still not detectable in vegetative hyphae or conidia. To assess the promoter activity of $U v$ Com 1, a 2.0-kb fragment of the UvComl promoter was cloned into the vector p3300neo-GFP. As a result, GFP signals were present in the cytoplasm of vegetative hyphae and conidia (Supplementary Fig. S3). These results suggested that the $U v$ Coml promoter may be constitutively active in $U$. virens. Because the UvCom1 protein contained two exons, we generated UvCom $1^{1-210}$-GFP and UvCom1211-659-GFP fusion construct p3300neo-GFP under the control of the RP27 promoter, and transformed them into the WT. The UvCom1211-659-GFP transformants showed strong fluorescent signals in the nuclei of conidia and vegetative hyphae (Fig. 5B), whereas no fluorescent signals were observed in the UvCom11-210_GFP transformants (Supplementary Fig. S3). These results suggested that full-length UvCom1 protein might affect the detection or production of the GFP signals, and that UvCom1211-659 determines the nuclear localization of UvCom1 protein.

Deletion of UvCom 1 might not affect the expression of genes associated with grain filling in rice. In previous studies,
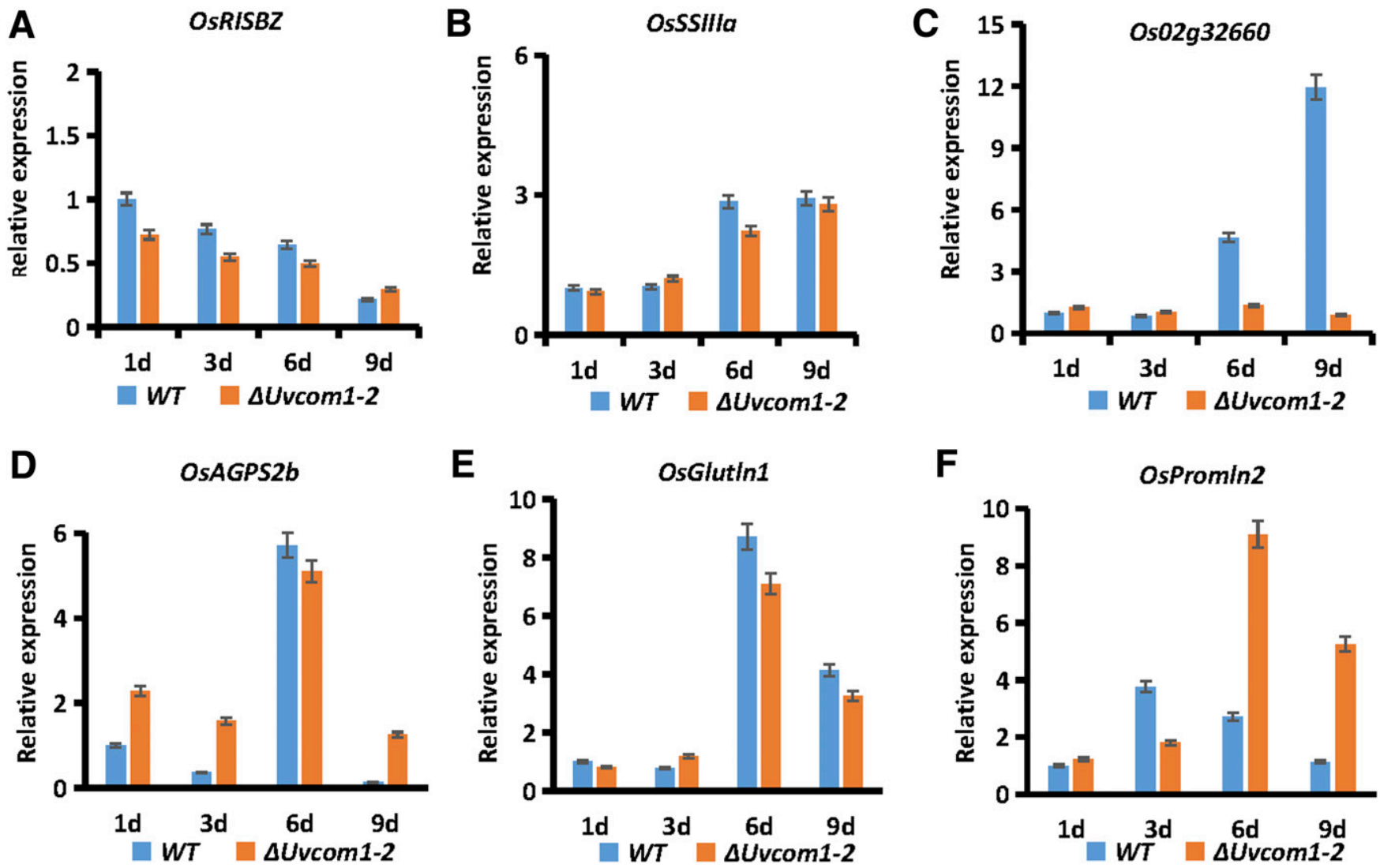

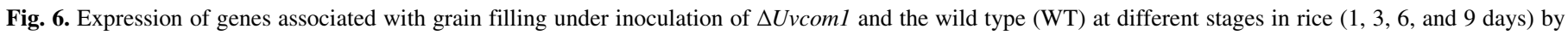

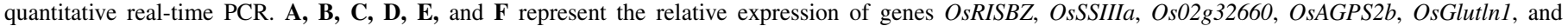
OsPromln2, respectively, in rice cultivar Wanxian-98. Error bars represent standard variations of three replicates in independent experiments. 
infection of $U$. virens induced the expression of genes associated with grain filling through imitated fertilization, and the rice provided large amounts of nutrients to the pathogen (Song et al. 2016). To determine whether UvCom 1 is involved in the regulation of genes related to grain filling in rice, the expression of rice genes associated with grain filling was determined after inoculation with the UvComl deletion mutant or WT. The results revealed that expression of most selected grain-filling-related genes, including OsRISBZ1, OsSSIIIa, OsAGPS $2 b$, and OsGlutln1, seems to be equal between the WT and $\Delta U v$ com 1-2 mutant, whereas OsO2g32660 and OsPromln 2 appear to be expressed much higher in the WT and $\Delta U v$ com1-2 mutant, respectively (Fig. 6A to F), indicating that the deletion of $U v C o m 1$ might not affect the expression of genes associated with grain filling, and failure of smut ball formation of $\Delta U v \operatorname{com} 1$ mutants is likely due to deficiency of pathogen development.
Genome-wide identification of genes regulated by UvCom1. In RNA-seq analysis, 625 DEGs were identified in the $\Delta$ Uvcom1-2 mutant, including 286 upregulated and 339 downregulated genes, based on the $\log _{2}$ ratio ( $\Delta U v$ coml -2 mutant/WT) values with twofold changes (Fig. 7A). Heatmaps were used to illustrate the differential expression of the genes detected in the WT and $\Delta U v$ com 1-2 mutant (Fig. 7B). In $U$. virens, 3,602 genes (43\%) were assigned to GO terms. The 625 DEGs were classified into 32 functional groups using GO analysis. Most of these DEGs were clustered into catalytic activity, metabolic process, single-organism process, and cellular process (Fig. 7C).

UvCom 1 played important roles in carbohydrate transport and metabolism, transmembrane transport, and transporter activity. In GO and KEGG enrichment analyses, a large number of DEGs were enriched in transmembrane transport, intracellular organelle part, and intrinsic and integral components of

\section{A}

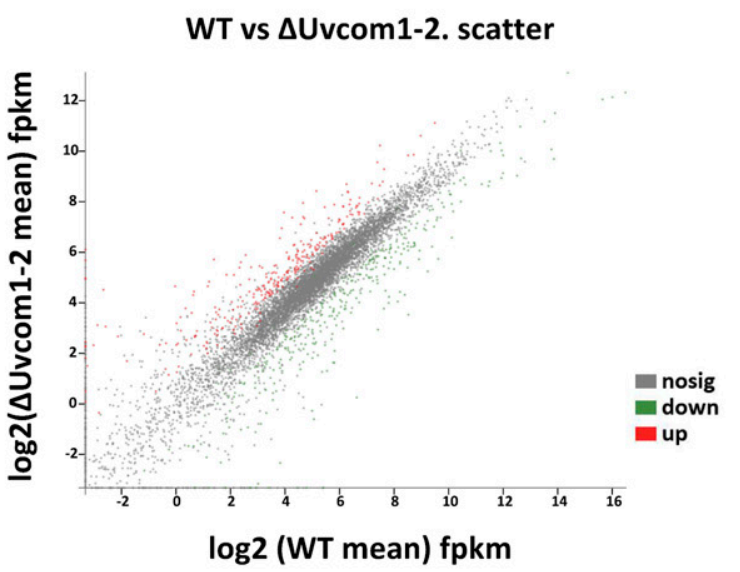

C

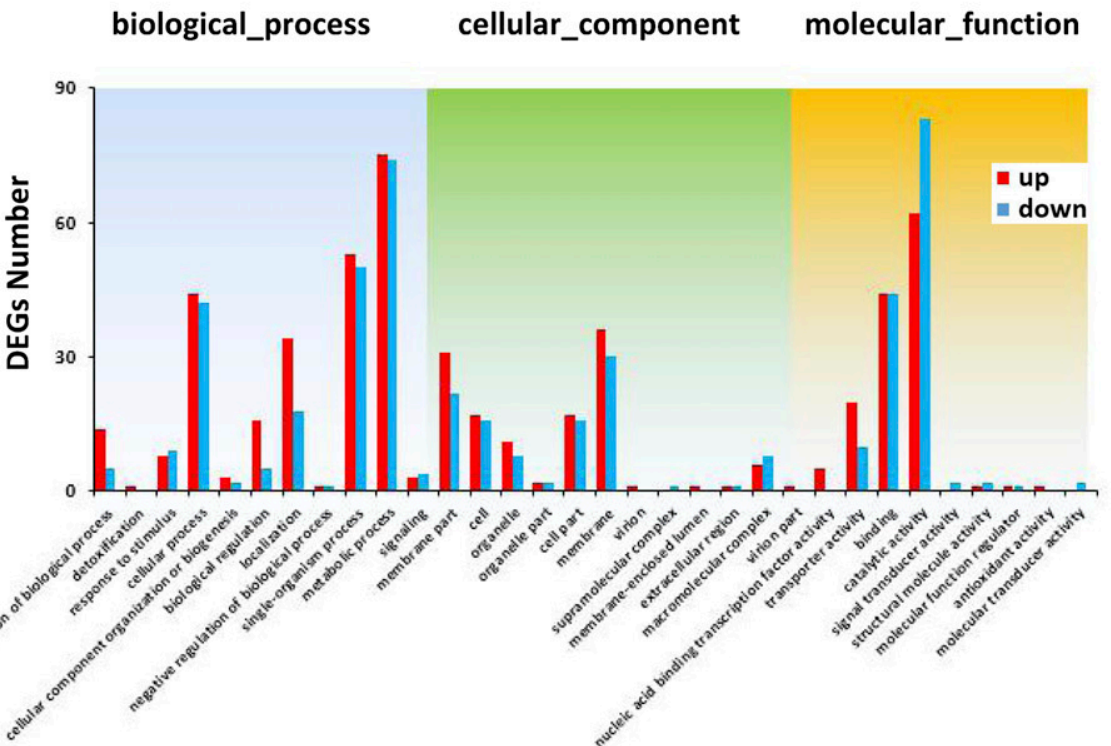

B
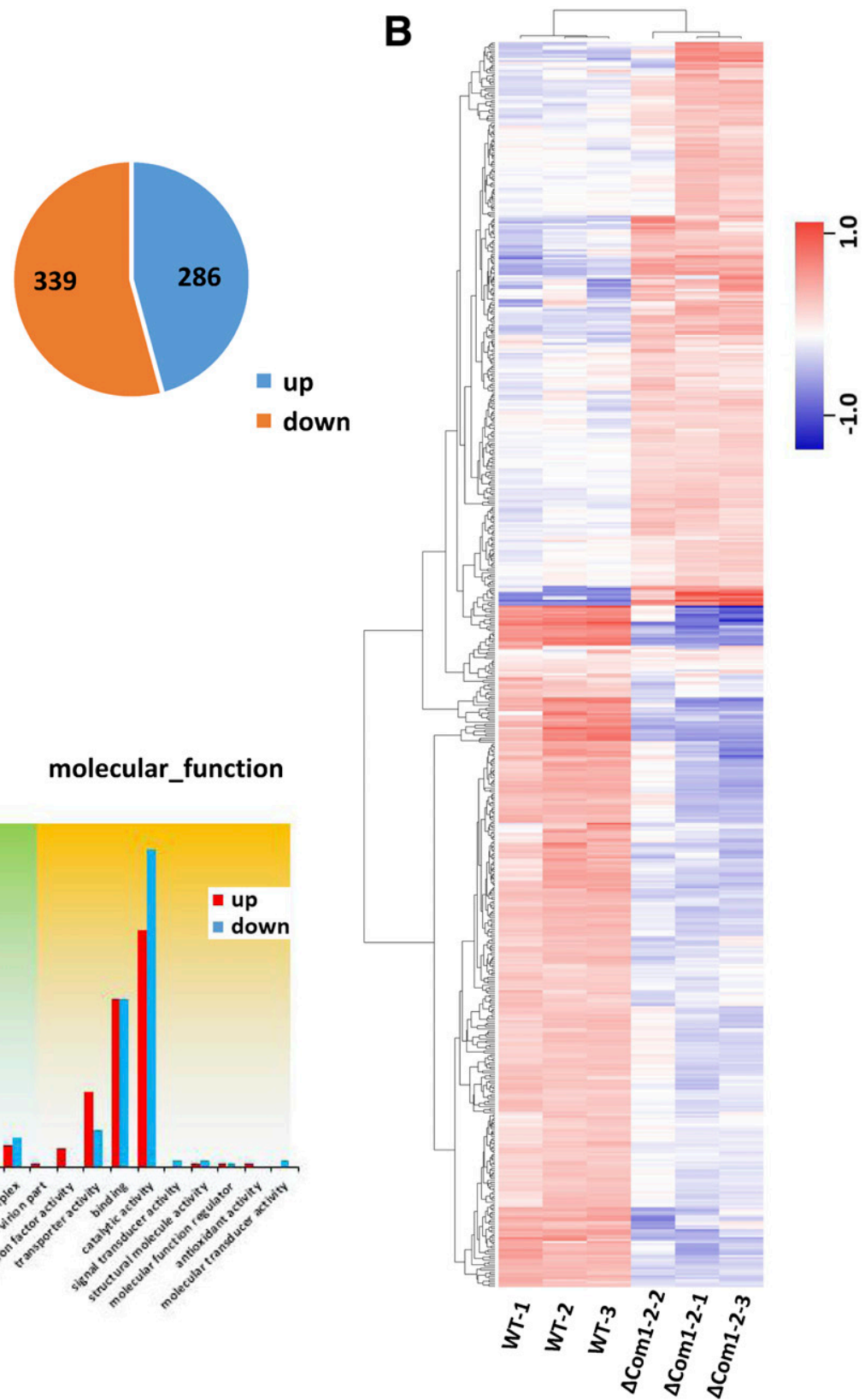

Fig. 7. Transcriptomic analysis to identify differentially expressed genes in $\Delta U v \operatorname{com} 1-2$ mutant and the wild type (WT). A, Number of genes with more than twofold changes in expression in $\Delta U v$ com 1-2. B, Heatmap of reads per kilobase of transcript per million mapped reads normalized transcripts that depicts the 625 differentially expressed genes in each pairwise comparison. C, Functional categorization of up- or downregulated genes in $\Delta U v \operatorname{com} 1-2$. DEGs $=$ differentially expressed genes. 


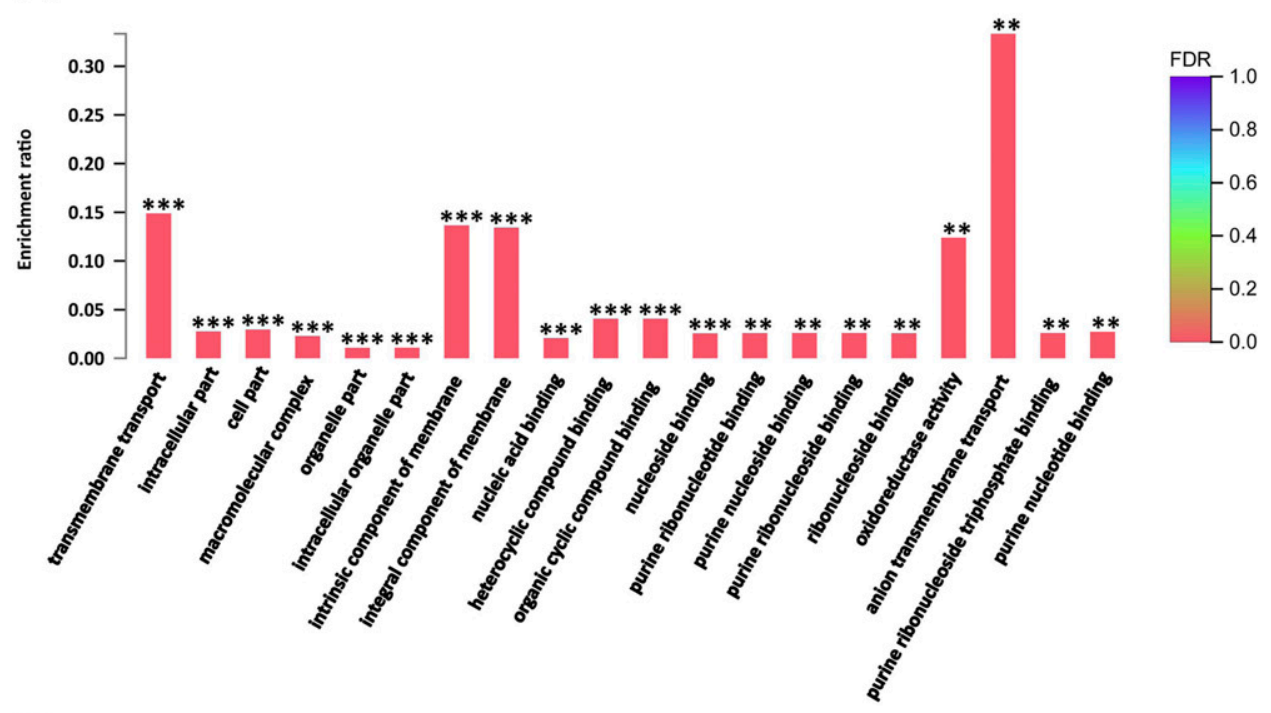

B

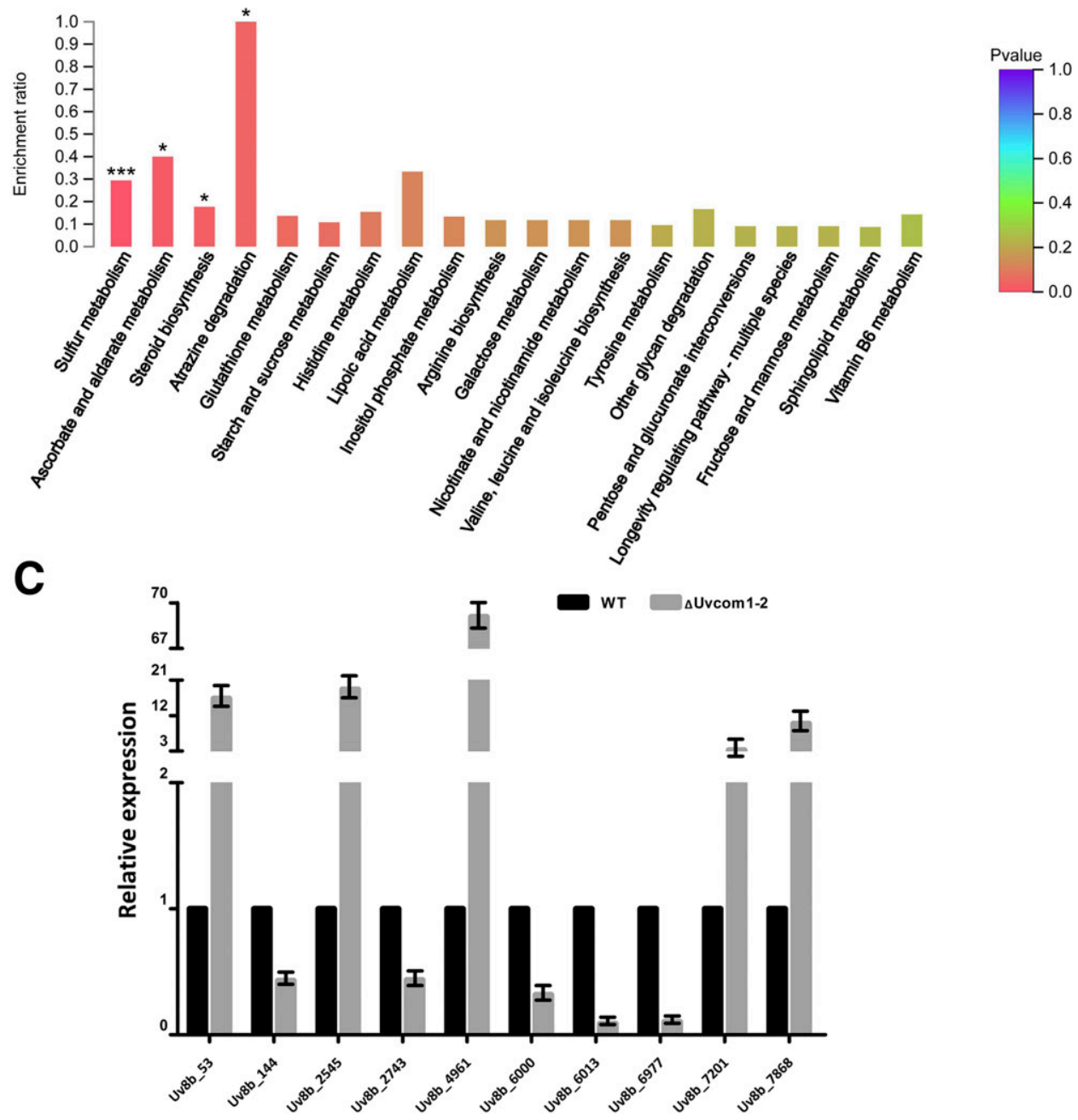

Fig. 8. Gene ontology (GO) and Kyoto Encyclopedia of Genes and Genomes (KEGG) functional annotations of unigenes in $\Delta U v c o m 1-2$ mutant and the wild-type (WT) transcriptome. The scatter diagram shows enrichment of differentially expressed genes (DEGs) in the signaling pathways. A, GO enrichment analysis. FDR = false discovery rate. B, KEGG enrichment analysis. The $\mathrm{X}$ axis represents enrichment ratio (enrichment ratio = amount of DEGs enriched in the pathway/amount of all genes in annotation gene set) and the Y axis represents the pathway $(P$ value $<0.05)$. C, Quantitative real-time PCR to validate differentially expressed genes related to transmembrane transporter. The relative abundance of the transcripts of each gene from $\Delta U v$ com $1-2$ was normalized by comparison with the gene from the WT (relative transcript $=1$ ). Uv8b_6013 = major facilitator superfamily (MFS) multidrug transporter, Uv8b_6977 = sugar transporter family protein, Uv8b_ $6000=$ putative metabolite transport protein, Uv8b_2743 = G-protein coupled receptor, Uv8b_144 = carboxylic acid transport protein, Uv8b_7201 = MFS phospholipid transporter Git1, Uv8b_7868 = urea active transporter, Uv8b_4961 = putative sodium/phosphate symporter, Uv8b_2545 = external NADHubiquinone oxidoreductase, and Uv8b_53 = oligopeptide transporter OPT-like protein. 

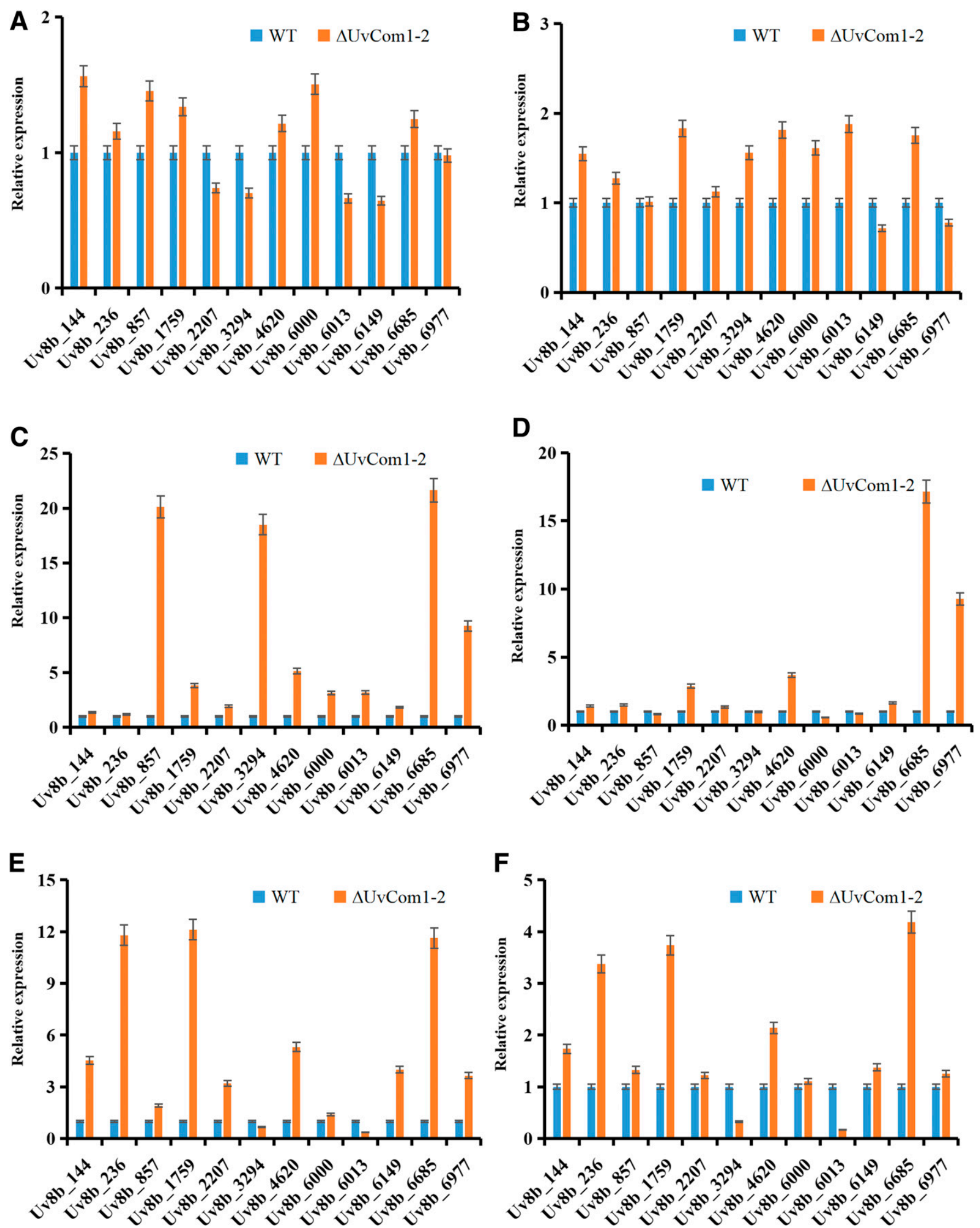

Fig. 9. Expression of 12 randomly selected genes associated with transmembrane transport of Ustilaginoidea virens under inoculation of the wild type (WT) and $\Delta$ Uvcom1-2 at different infection stages in rice by quantitative real-time PCR: A, 1 day postinoculation (dpi;) B, 3 dpi; C, 6 dpi; D, 9 dpi; E, 13 dpi; and F, 20 dpi. Error bars represent standard variations of three replicates in independent experiments. 
membrane pathways (Fig. 8A and B), which are related to carbohydrate transport and metabolism, transmembrane transport, and transporter activity (Supplementary Tables S4, S5, and S6). We found that many DEGs belonged to the major facilitator superfamily (MFS) (UV8b_6013, UV8b_6977, UV8b_6000, UV8b_6685, UV8b_144, UV8b_6649, UV8b_236, UV8b_3294, UV8b_4620, UV8b_2207, UV8b_1759, UV8b_7201, UV8b_857), inorganic ion transport and metabolism (UV8b_4845, UV8b_654, UV8b_2367, UV8b_2511, UV8b_5548, UV8b_8112, UV8b_366, UV8b_5308, and UV8b_4961), and amino acid transport and metabolism (UV8b_1682, UV8b_121, UV8b_5087, UV8b_6984, UV8b_6592, UV8b_8057, and UV8b_7868). To confirm the gene expression patterns, we selected 10 genes for qRT-PCR analyses. The results showed that the expression pattern of each upregulated or downregulated gene was consistent with that in the RNA-seq data (Fig. 8C). To further validate the expression patterns of transportrelated genes between the WT and $\Delta U v$ com $1-2$ mutant in planta, we randomly selected 12 genes related to transmembrane transport for qRT-PCR, and expressions of these genes were significantly upregulated or downregulated at different infection stages $(1,3,6$, 9, 13, or 20 dpi) (Fig. 9) $(P<0.05)$. These results indicated that UvCom1 plays an important role in transmembrane transport.

\section{DISCUSSION}

Com1, which is specifically conserved in species of Basidiomycetes and Ascomycetes, has only been previously identified in M. oryzae (Bhadauria et al. 2010; Yang et al. 2010), and little is known about its function in fungi. In this study, deletion of $U v C o m 1$ caused a lower growth rate of $U$. virens, which is different from the observations in the $\Delta$ Mocoml mutants. In M. oryzae, $\Delta$ Mocoml mutants showed a morphology of longer and narrower conidia than the WT (Yang et al. 2010) but, in $U$. virens, deletion of $U v C o m 1$ did not affect the conidial morphology. Inhibition of conidiation was found in the $\Delta U v \operatorname{com} 1$ mutants, which is similar to the results obtained in $M$. oryzae. Our results indicated that UvComl negatively regulates the responses of the $U$. virens to oxidative and osmotic stress and cell wall integrity. In RNA-seq data, many membrane transporters were induced significantly in the UvComl deletion mutant, and the alteration of membrane transport in the mutant might play a role in its negative response to these various stresses. However, limited work has been done to investigate the relationships between membrane transporters and oxidative, osmotic, or cell wall stress responses in fungi. Biological functions of the upregulated transporters could be elucidated in the future. Transcription factor $S K N 7$ has been reported to be involved in the regulation of stress-related genes in $F$. graminearum (Jiang et al. 2015), and GPDI and CTT1 were found to regulate the response to osmotic stress in different fungi (Albertyn et al. 1994; Schüller et al. 1994). Our RNA-seq data showed that these three genes were upregulated in UvComl deletion mutants, suggesting that the deletion of $U v C o m 1$ affects the expression of other regulators of stress response. Overall, our results indicate that Com 1 homologs play species-dependent roles in morphogenesis of different fungi but have conserved functions in conidiation.

In this study, GFP-UvCom1 or UvCom1-GFP fusion constructs under the control of RP27 or its native promoter were generated and transformed into the WT but no GFP signals were observed in either conidia or vegetative hyphae under confocal microscopy. Similar results were obtained in $M$. oryzae (Yang et al. 2010). We hypothesize that the full-length UvCom1 protein might have an adverse effect on the fluorescence of the GFP fusion protein. To test this hypothesis, UvCom11-210-GFP or UvCom1211-659-GFP fusion constructs were transformed into the WT. As expected, UvCom1211-659-GFP fusion construct transformants showed strong fluorescent signals in the nuclei of conidia and vegetative hyphae. In $M$. oryzae, the PPVKRPRE or PLAKKFK sequences could direct the nuclear localization of Mocom1 $1^{335-613}$-GFP fusion proteins
(Yang et al. 2010). By alignment, the PPVKRPRE sequence was also found to exist in the UvCom1 protein sequence, and it might also direct the nuclear localization in $U$. virens.

The $\Delta U v \operatorname{com} 1$ mutants could normally infect rice filaments, stigmas, and styles, and establish a preliminary nutritional relationship with rice spikelets. However, the mutants might have lost the ability to fully utilize the nutrition from rice, and the mycelia in the spikelets could not expand to form typical false smut balls. We also tested the expression of genes related to grain filling after infection by the WT or $\Delta U v$ coml mutants. The results showed that all of these genes could be expressed at different stages of infection, indicating that rice grain filling was not suppressed by inoculation with $\Delta$ Uvcoml mutants. We hypothesized that the deletion of UvCom1 might lead to the loss of sugar transport for the fungus. RNA-seq data showed that many genes enriched in membrane transport pathways were downregulated in the $\Delta U$ vcom 1 mutant. In Saccharomyces cerevisiae, monosaccharide uptake is mediated by transporters belonging to the major facilitator superfamily (Young et al. 2013). Biotrophic phytopathogenic fungi might infect plants to obtain nutrients by redirecting carbon and nitrogen resources. Among these MFS genes, a sugar transporter family protein (UV8b_6977) was significantly downregulated in RNA-seq data. We examined the gene expression profile at different developmental stages of $U$. virens during pathogenesis by qRT-PCR, and found that the expression of UV8b_6977 was significantly increased from the stage of mycelial expansion to the formation of smut balls in rice spikelets (Supplementary Fig. S4). The functional link between UvCom1 and UV8b_6977 in fungal infection needs to be confirmed.

RNA interference (RNAi) is a conserved regulatory mechanism of gene expression and has become a powerful tool to silence genes (Waterhouse and Fusaro 2006). RNAi starts with the initial processing or cleavage of a precursor double-stranded RNA (dsRNA) into short (21 to 25 nucleotides) small-interfering RNA or microRNA duplexes by Dicer (Baulcombe 2004; Hamilton and Baulcombe 1999). In planta, the expression of dsRNAs was recently shown to induce host plant-induced gene silencing (HIGS) in fungal cells (Koch et al. 2013). HIGS has been used to reduce the severity of fungal diseases in wheat, barley, and banana (Cheng et al. 2015; Ghag et al. 2014; Nowara et al. 2010). In wheat, HIGS of $\mathrm{FgChs}$ and $\mathrm{FgCYP} 51$ could enhance resistance to $F$. graminearum (Cheng et al. 2015; Koch et al. 2013). Silencing of fungal glucanosyltransferase genes enhanced resistance to Blumeria graminis (Nowara et al. 2010). Com1 is conserved in fungi and is not found in plants or animals, including humans. As a virulence factor of phytopathogens, this protein could be used in the future as a specific molecular target for disease control. We speculate that $U v C o m l$, which is essential for $U$. virens development and infection, is possibly a feasible RNAi target for promoting the resistance of rice plants against rice false smut.

Overall, it can be speculated that the $U v \operatorname{Com} 1$ plays a key role in regulating transmembrane transport by facilitating the utilization of large quantities of nutrients to form rice false smut balls. This is the first hypothesized mechanism of the involvement of a Coml gene in the virulence of a biotrophic phytopathogen. More evidence is still needed to elucidate the regulatory role of $U v \mathrm{Com} 1$ in transmembrane transport.

\section{ACKNOWLEDGMENTS}

We thank Wenxian Sun at China Agricultural University for providing the pGKO gene deletion vectors.

\section{LITERATURE CITED}

Albertyn, J., Hohmann, S., Thevelein, J. M., and Prior, B. A. 1994. GPD1, which encodes glycerol-3-phosphate dehydrogenase, is essential for growth under osmotic stress in Saccharomyces cerevisiae, and its expression is regulated by the high-osmolarity glycerol response pathway. Mol. Cell. Biol. 14:4135-4144. 
Ashizawa, T., Takahashi, M., Arai, M., and Arie, T. 2012. Rice false smut pathogen, Ustilaginoidea virens, invades through small gap at the apex of a rice spikelet before heading. J. Gen. Plant Pathol. 78:255-259.

Baulcombe, D. 2004. RNA silencing in plants. Nature 431:356-363.

Bhadauria, V., Wang, L. X., and Peng, Y. L. 2010. Proteomic changes associated with deletion of the Magnaporthe oryzae conidial morphologyregulating gene COM1. Biol. Direct 5:61.

Brooks, S. A., Anders, M. M., and Yeater, K. M. 2010. Effect of furrow irrigation on the severity of false smut in susceptible rice varieties. Plant Dis. 94:570-574

Cheng, W., Song, X. S., Li, H. P., Cao, L. H., Sun, K., Qiu, X. L., Xu, Y. B., Yang, P., Huang, T., Zhang, J. B., Qu, B., and Liao, Y. C. 2015. Hostinduced gene silencing of an essential chitin synthase gene confers durable resistance to Fusarium head blight and seedling blight in wheat. Plant Biotechnol. J. 13:1335-1345.

Fan, J., Guo, X. Y., Li, L., Huang, F., Sun, W. X., Li, Y., Huang, Y. Y., Xu, Y. J., Shi, J., Lei, Y., Zheng, A. P., and Wang, W. M. 2015. Infection of Ustilaginoidea virens intercepts rice seed formation but activates grainfilling-related genes. J. Integr. Plant Biol. 57:577-590.

Ghag, S. B., Shekhawat, U. K. S., and Ganapathi, T. R. 2014. Host-induced post-transcriptional hairpin RNA-mediated gene silencing of vital fungal genes confers efficient resistance against Fusarium wilt in banana. Plant Biotechnol. J. 12:541-553.

Guo, X. Y., Li, Y., Fan, J., Li, L., Huang, F., and Wang, W. M. 2012. Progress in the study of false smut disease in rice. J. Agric. Sci. Technol. 2:1211-1217.

Hamilton, A. J., and Baulcombe, D. C. 1999. A species of small antisense RNA in post transcriptional gene silencing in plants. Science 286:950-952.

Jia, Q., Lv, B., Guo, M. Y., Luo, C. X., Zheng, L., Hsiang, T., and Huang, J. B. 2015. Effect of rice growth stage, temperature, relative humidity and wetness duration on infection of rice panicles by Villosiclava virens. Eur. J. Plant Pathol. 141:15-25.

Jiang, C., Zhang, S., Zhang, Q., Tao, Y., Wang, C., and Xu, J. R. 2015. FgSKN7 and FgATF1 have overlapping functions in ascosporogenesis, pathogenesis and stress responses in Fusarium graminearum. Environ. Microbiol. 17:1245-1260.

Jiang, Z., Jiang, Z., Jiang, Z., and Zhang, G. 2009. Effect of false smut ball number on yield of rice. North. Rice 39:53-54.

Koch, A., Kumar, N., Weber, L., Keller, H., Imani, J., and Kogel, K. H. 2013. Host-induced gene silencing of cytochrome P450 lanosterol C14 $\alpha$ demethylase-encoding genes confers strong resistance to Fusarium species. Proc. Natl. Acad. Sci. U.S.A. 110:19324-19329.

Koiso, Y., Li, Y., Iwasaki, S., Hanaoka, K., Kobayashi, T., Sonoda, R., Fujita, Y., Yaegashi, H., and Sato, Z. 1994. Ustiloxins, antimitotic cyclic peptides from false smut balls on rice panicles caused by Ustilaginoidea virens. J. Antibiot. 47:765-773

Li, B., and Dewey, C. N. 2011. RSEM: Accurate transcript quantification from RNA-Seq data with or without a reference genome. BMC Bioinf. 12: 323.

Lv, B., Zheng, L., Liu, H., Tang, J. T., Hsiang, T., and Huang, J. B. 2016. Use of random T-DNA mutagenesis in identification of gene UvPRO1, a regulator of conidiation, stress response, and virulence in Ustilaginoidea virens. Front. Microbiol. 7:2086.

Nakamura, K., Izumiyama, N., Ohtsubo, K., Koiso, Y., Iwasaki, S., Sonoda, R., Fujita, Y., Yaegashi, H., and Sato, Z. 1994. "Lupinosis"-like lesions in mice caused by ustiloxin, produced by Ustilaginoidea virens: A morphological study. Nat. Toxins 2:22-28.

Nowara, D., Gay, A., Lacomme, C., Shaw, J., Ridout, C., Douchkov, D., Hensel, G., Kumlehn, J., and Schweizer, P. 2010. HIGS: Host-induced gene silencing in the obligate biotrophic fungal pathogen Blumeria graminis. Plant Cell 22:3130-3141.

Qiu, J., Meng, S., Deng, Y., Huang, S., and Kou, Y. 2019. Ustilaginoidea virens: A fungus infects rice flower and threats world rice production. Rice Sci. 26:199-206.

Robinson, M. D., McCarthy, D. J., and Smyth, G. K. 2010. edgeR: A Bioconductor package for differential expression analysis of digital gene expression data. Bioinformatics 26:139-140.

Schüller, C., Brewster, J. L., Alexander, M. R., Gustin, M. C., and Ruis, H. 1994. The HOG pathway controls osmotic regulation of transcription via the stress response element (STRE) of the Saccharomyces cerevisiae CTT1 gene. EMBO J. 13:4382-4389.

Song, J. H., Wei, W., Lv, B., Lin, Y., Yin, W. X., Peng, Y. L., Schnabel, G., Huang, J. B., Jiang, D. H., and Luo, C. X. 2016. Rice false smut fungus hijacks the rice nutrients supply by blocking and mimicking the fertilization of rice ovary. Environ. Microbiol. 18:3840-3849.

Tang, Y. X., Jin, J., Hu, D. W., Yong, M. L., Xu, Y., and He, L. P. 2013. Elucidation of the infection process of Ustilaginoidea virens (teleomorph: Villosiclava virens) in rice spikelets. Plant Pathol. 62:1-8.

Trapnell, C., Pachter, L., and Salzberg, S. L. 2009. TopHat: Discovering splice junctions with RNA-Seq. Bioinformatics 25:1105-1111.

Waterhouse, P. M., and Fusaro, A. F. 2006. Viruses face a double defense by plant small RNAs. Science 313:54-55.

Xie, C., Mao, X., Huang, J., Ding, Y., Wu, J., and Dong, S. 2011. KOBAS 2.0: A web server for annotation and identification of enriched pathways and diseases. Nucleic Acids Res. 39:W316-W322.

Yang, J., Zhao, X. Y., Sun, J., Kang, Z. S., Ding, S. L., Xu, J. R., and Peng, Y. L. 2010. A novel protein Com1 is required for normal conidium morphology and full virulence in Magnaporthe oryzae. Mol. Plant-Microbe Interact. 23:112-123.

Yang, L., Chen, L., Xu, J., Liu, J., and Ding, K. 2012. Estimation of yield loss caused by rice false smut. J. Anhui Agric. Univ. 39:474-477.

Young, E. M., Tong, A., Bui, H., Spofford, C., and Alper, H. S. 2013. Rewiring yeast sugar transporter preference through modifying a conserved protein motif. Proc. Natl. Acad. Sci. U.S.A. 1:131-136.

Yu, J. J., Yu, M. N., Song, T. Q., Cao, H. J., Yong, M. L., Qi, Z. Q., Du, Y., Zhang, R. S., Yin, X. L., and Liu, Y. F. 2019. A homeobox transcription factor $U v H O X 2$ regulates chlamydospore formation, conidiogenesis and pathogenicity in Ustilaginoidea virens. Front. Microbiol. 10:1071.

Zhang, Y., Zhang, K., Fang, A. F., Han, Y. Q., Yang, J., Xue, M. F., Bao, J. D., Hu, D. W., Zhou, B., Sun, X. Y., Li, S. J., Wen, M., Yao, N., Ma, L. J., Liu, Y. F., Zhang, M., Huang, F., Luo, C. X., Zhou, L. G., Li, J. Q., Chen, Z. Y., Miao, J. K., Wang, S., Lai, J. S., Xu, J. R., Hsiang, T., Peng, Y. L., and Sun, W. X. 2014. Specific adaptation of Ustilaginoidea virens in occupying host florets revealed by comparative and functional genomics. Nat. Commun. 5: 3849 .

Zhou, L., Lu, S., Shan, T., Wang, P., Sun, W., Chen, Z., and Wang, S. 2012. Chemistry and biology of mycotoxins from rice false smut pathogen. Pages 109-130 in: Mycotoxins: Properties, Applications and Hazards. B. J. Melborn and J. C. Greene, eds. Nova Science Publishers, New York, NY, U.S.A. 